\title{
Case Study on Using the User-Centric-Backhaul Scheme to Unlock the Realistic Backhaul
}

\author{
Mona Jaber ${ }^{1}$, Muhammad Ali Imran ${ }^{1,2}$, Anvar Tukmanov ${ }^{3}$, Andy Sutton ${ }^{4}$, and Rahim Tafazolli ${ }^{1}$ \\ ${ }^{1}$ Institute for Communication Systems, Home of 5G Innovation Centre, University of Surrey, Guildford, GU2 7XH, UK \\ Email: $\{$ m.jaber, m.imran, r.tafazolli\}@ surrey.ac.uk \\ 2 School of Engineering, University of Glasgow, Glasgow, G12 8QQ, UK Email: Muhammad.Imran@glasgow.ac.uk \\ ${ }^{3}$ BT Research and Innovation, Adastral Park, Ipswich, IP5 3RE, UK Email: anvar.tukmanov@bt.com \\ ${ }^{4}$ School of Computing, Science and Engineering at the University of Salford, Salford, M5 4WT, UK Email: andy.sutton@ee.co.uk
}

\begin{abstract}
The fifth generation of mobile networks (5G) is maturing fast and the target year 2020 is around the corner. However, the realistic backhaul network may not be ready for $5 \mathrm{G}$ arrival as it is likely to converge to $5 \mathrm{G}$ requirements at a slower pace than the radio counterpart. In this work, we develop a method that identifies pertinent backhaul upgrade stages that are ranked based on their associated cost. First, the User-centric-backhaul (UCB) scheme is employed to reveal the bottlenecks of the incumbent backhaul network, as perceived by users and holistic network. A multi-hop hybrid backhaul modelling framework is then employed to quantify possible rectifications that would deliver the highest improvement at the lowest cost. These are implemented and the results are verified following another usage of UCB. A case study is presented that demonstrates the strength of this method in enabling an effective and cost efficient evolution road map towards the 5G backhaul.
\end{abstract}

\section{INTRODUCTION}

In a recent survey, about $60 \%$ of participating operators said they plan to trial $5 \mathrm{G}$ by $2018,32 \%$ plan to launch pre-standard $5 \mathrm{G}$, and $17 \%$ predict that $5 \mathrm{G}$-related costs will be included in the capital expenditure plan of year 2018 [1]. Indeed, $5 \mathrm{G}$ is no longer a futuristic vision but has become today's reality and demands an imminent and efficient approach to tackle the related deployment challenges. The backhaul section of the network is not the least challenging. Recent research has shown that the performance evolution of the current realistic backhaul to the $5 \mathrm{G}$ grade of service is a lengthy and costly process. Only $3 \%$ of the operators are considering testing the backhaul network in the first trials where $68 \%$ have the radio network as priority [1]. Therefore, the backhaul will undergo slower evolution towards ubiquitous $5 \mathrm{G}$ performance. Theoretically, fibre is believed to be the ultimate backhaul solution, however, it is also known to be highly impractical for network-wide deployment due to the cumbersome and very slow process of laying new fibre in urban areas. Consequently, a multi-hop and hybrid backhaul network would serve early stages of $5 \mathrm{G}$ and would offer a diverse range of characteristics based on the topology and technology mix. 5G users (devices and applications) have very different quality needs, hence the network needs to adapt to deliver user-centric quality with minimum incurred signalling overhead [2]. Indeed, authors in [1] say that: " $5 \mathrm{G}$ needs to be a chameleon technology that can adapt to differing demands of wireless services whether to support high bandwidth, low latency, bursty traffic, ultra-reliable services, or a combination of these capabilities".

The User-centric Backhaul (UCB) is the state-of-the-art solution for intelligent user-cell-backhaul association that is context-aware, radio-network-aware, and backhaul-aware, and as such, affords the network the "chameleon-like" characteristics [3]. It was demonstrated in [3], that the UCB reveals the hard limits of the network that cannot be circumvented by intelligent user-cell-backhaul matching. It is vital for operators deploying $5 \mathrm{G}$ networks to determine the weak aspects of the incumbent backhaul and identify pertinent solutions that yield the highest benefits at the minimum cost. In this work, we propose the first approach to a cost-effective and quality-aware backhaul upgrade using the UCB in a case-study.

A realistic dense network of small cells is created based on elements of stochastic geometry, as in [4]. The performance of the multi-hop backhaul links is generated based on technology-specific analytical models and according to the topology. The UCB is first used to gauge the gap between the available and required network performance by looking at two attributes: Throughput and Latency. The performance modelling approach in [4] is then used to obtain alternative solutions for reducing this gap. These alternatives are then ranked according to their incurred gains and total cost of ownership (TCO). Two solutions are shortlisted and are next implemented in the simulated network and their effectiveness is measured using the UCB. The results presented in the paper advocate the strength of this approach in bridging the backhaul performance gap and improving the user-centric quality.

There are several works that tackle the intelligent user-cellbackhaul association problem (e.g., [5], [6]). Others works propose analytical models for the backhaul or end-to-end network performance modelling (e.g., [7], [8]). However, to the best of our knowledge, this is the first work that proposes an end-to-end methodology that measures the gap, generates rectification solutions, ranks their corresponding cost-effectiveness, which is then validated in simulations. The rest of the paper is organised as follows. The system model is first presented in Section II. In Section III, we describe the case study initial conditions and UCB results and interpretations. The methodology of identifying possible solutions is described in Section IV as well as the TCO model and corresponding ranking. The paper is concluded in Section V. Please note that the notation $\mathrm{E}[x]$ indicates the expectation of the variable $x$ and $\mathrm{E}[f(x, y) \mid x]$ is the expectation of the function $f$ conditioned on the value $x$.

\section{SySTEM MODEL}

We consider a dense network of small cells which have identical radio characteristic. Each cell connects back to a backhaul aggregation point using one of three possible transport technologies: VDSL2 ${ }^{1}$ (copper-based), microwave (28 GHz), and 10G-PON (10 Gbps). Aggregation points are linked to the core network through a fibre-based backhaul. The last mile is thus the network throughput bottleneck. The described system is simulated in Matlab as a multi-layered network in which users belong to Layer 0 , small cells to Layer 1 and aggregation points to Layer 2. Each of these layers is described

\footnotetext{
${ }^{1}$ Very-high-bit-rate digital subscriber line 2
} 


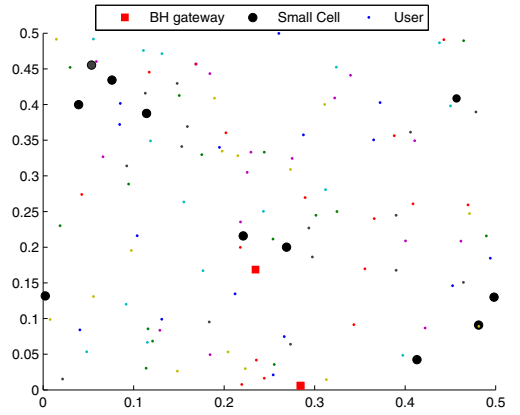

Fig. 1. Example of network model.

by a Poisson Point Process (PPP) $\Phi_{l}$ with density $\lambda_{l}$ for $l \in[0,1,2]$. The link between Layers 0 and 1 is an LTE-based radio interface. The link between Layers 1 and 2 is the last mile backhaul network which consists of $p_{v}$ share of VDSL2, $p_{m}$ of microwave, and $p_{f}$ of fibre links, such that $p_{v}+p_{m}+p_{f}=1$. An example network is shown in Figure 1.

\section{A. Monte Carlo simulations}

In each Monte Carlo run, an instance of the described network model is generated with the following three parameters: (i) number, location, and quality requirements of users, (ii) number, location, and type of last mile of small cells, (iii) number and location of aggregation points. The distance between any cell in $\Phi_{1}$ and any gateway in $\Phi_{2}$ is then computed and the shortest is selected. Consequently, the capacity and latency expectations of each cell's last mile are derived. In each iteration, the users parameters are updated and the corresponding metrics stored. The UCB consists of dynamically setting two bias factors for each cell that correspond to the capacity and latency attributes, respectively. A higher bias value indicates better performance and vice-versa. Let $u$ denote a user in Layer 0, each user $u$ associates two sets of parameters that are randomly generated: $\left\{Q_{u, c}, Q_{u, l}\right\}$ and $\left\{\omega_{u, c}, \omega_{u, l}\right\}$. The first set indicates the target values of throughput and latency, respectively. The second represents the weights associates with each attribute. The UCB endeavours to find the optimum setting of each bias factor in each cell is such a way that maximise the users' satisfaction and the network throughput. The users' and network' key performance indicators (KPIs) that are used to gear this process are described next.

\section{B. Users' and network KPIs}

Assessing the effectiveness of a user-cell-backhaul scheme may only be performed after identifying adequate and representative metrics. Traditionally, service-based metrics have been used to assess the network's performance, such as peak data rates, coverage, and spectral efficiency, termed Quality of Service or QoS. However, 5G networks are about the "Always Sufficient Rate to give Users the perception of Infinite Capacity", thus the key metric should gauge subjective users' perception of the quality and not objective QoS. ITU-T G.114 recommends a maximum one-way delay of $150 \mathrm{msec}$ for Voice-over-IP beyond which the degradation may be discerned. On the other hand, delay requirements for online gaming differ greatly, depending on the game played (e.g., Battlefield 2 and MMORPGs ${ }^{3}$ ). "Elite" performance of a maximum delay of $50 \mathrm{msec}$ is recommended for intense first-person shooters games such as Battlefield 2. Other real-time strategy games, e.g., MMORPGs,

\footnotetext{
${ }^{2}$ http://www.surrey.ac.uk/5gic/about/5gic-vision

${ }^{3}$ http://www.mmorpg.com/games-list and http://www2.ea.com/battlefield-2
}

can run with a delay of $150 \mathrm{~ms}$ [9]. From a user-centric point of view, a delay of $100 \mathrm{msec}$ (QoS value) leads to excellent quality of experience (QoE) for this game or voice-over-IP. The same QoS value would result in an unacceptable QoE for a Battlefield 2 player.

To this end, $5 \mathrm{G}$ optimisation demands novel metrics that are centered on users' QoE and are context-aware [10]. The mean opinion score (MOS) is the most popular indicator for measuring such perceived media quality. Nonetheless, gathering sufficient scores from users, referred to as subjective MOS, is often a delicate task that is time consuming and costly. Objective MOS methods are automated alternatives that may be calibrated to reflect the quality reported by the subjective MOS and can readily be employed in optimisation schemes of cellular systems [11]. One 5G challenge is to define a context-agnostic mapping between QoE and easy-to-use QoS, which is beyond the scope of this work. In this case study, we assume that the QoE-to-QoS mapping is a given for all 5G use-cases. For each use-case, we associate a set of required QoS parameters with corresponding weights. It is assumed that these QoS and weights have been tuned to reflect the target QoE of the use-cases. These are compared to the achieved QoS, i.e. delivered by the network. The metric we propose to measure the users' satisfaction gauges the gaps between each of the target and delivered QoS parameters, and is affected by the corresponding weight. Let $\left\{Q_{u, c}^{\prime}, Q_{u, l}^{\prime}\right\}$ represent the measured QoS values delivered by the network to user $u$. We define the level of dissatisfaction $\left(\hat{Q}_{u, q}\right)$ of a user $u$ with respect to quality attribute $q=\{c$ (capacilty), $l$ (latency) $\}$ as the ratio of the gap $\left(Q_{u, q}^{\prime}-Q_{u, q}\right)$ to the target $\operatorname{QoS}\left(Q_{u, q}\right)$. The cumulative dissatisfaction of users who associate a high weight to the given quality is $\hat{Q}_{q}$, shown below. We also estimate the cumulative weighted users' dissatisfaction $\overline{Q_{q}}$ for both types of users. It should be noted that the measured $Q_{u, q}^{\prime}$ is clamped to the target value, i.e., it is not beneficial to exceed it nor does it compensate for quality shortage of other users.

$$
\begin{gathered}
\hat{Q}_{q} \triangleq \frac{1}{\lambda_{0}} \sum_{u}\left|\frac{Q_{u, q}^{\prime}-Q_{u, q}}{Q_{u, q}}\right| \mid \omega_{u, q}=\text { High } \\
\overline{Q_{q}} \triangleq \frac{1}{\lambda_{0}} \sum_{u} \omega_{u, q} \times \hat{Q}_{u, q}, \forall q \in\{c, l\}
\end{gathered}
$$

On the other hand, maximising the infrastructure usage efficiency remains an operator's prime target. To this end, it is desirable for a novel backhaul scheme to maximise the backhaul load without overloading. By doing so, the total system throughput is also maximised and the best utilisation of the infrastructure is achieved. Consequently, a key network-centric QoS metric is the total system throughput $T_{\text {tot }}$ and the rate of unsatisfied users $O_{c}$ with respect to capacity and $O_{l}$ to latency.

\section{CASE STUDY}

The case study is built based on the system model described in the previous section with parameters as defined in Table III, which constitute the baseline scenario. The capacity and latency modelling of the backhaul network are described in Section III-A and the cost model in Section III-B.

\section{A. Performance models}

The performance modelling is focused on the backhaul network as the radio access is purposely over-dimensioned. Based on the work in [4], the capacity of a multi-hop hybrid backhaul is determined by the hop that is the most limiting: the last mile. The latency, on the other hand, is the aggregate effect of delay occurring on all hops. We consider that the backhaul network between the small cell backhaul gateway and the core network has a cumulative mean delay of $10 \mathrm{msec}$. The parameters employed to model the VDSL2 and microwave links' capacity and latency are listed in Table III-A. The expression characterising the expected capacity of a VDSL2 hop for $r$ 
TABLE I

SIMULATION PARAMETERS.

\begin{tabular}{|c|c|l|}
\hline Parameter & Value & Remark \\
\hline$\lambda_{0}$ & 400 users $/ \mathrm{km}^{2}$ & Density of $\Phi_{0}$ PPP representing users. \\
\hline$\lambda_{1}$ & 40 cells $/ \mathrm{km}^{2}$ & Density of $\Phi_{1}$ PPP representing small cells. \\
\hline$\lambda_{2}$ & 4 gateways $/ \mathrm{km}^{2}$ & Density of $\Phi_{2}$ PPP representing backhaul aggregation points. \\
\hline$Q_{c}$ & {$[0,5] \mathrm{dB}$} & $50 \%$ of users are randomly allocated a low target SINR and $50 \%$ a high target. \\
\hline$Q_{l}$ & {$[30,100] \mathrm{msec}$} & $50 \%$ of users are randomly allocated a low target latency and $50 \%$ a high target. \\
\hline$\omega_{c}$ & {$[0.1,0.9] \mathrm{dB}$} & $50 \%$ of users are randomly allocated a low weight to SINR target and 50\% a high weight. \\
\hline$\omega_{l}$ & {$[0.1,0.9] \mathrm{dB}$} & $50 \%$ of users are randomly allocated a low weight to latency target and 50\% a high weight. \\
\hline$p_{v}$ & $40 \%$ & $40 \%$ of last mile links are randomly allocated VDSL2 technology. \\
\hline$p_{m}$ & $30 \%$ & $30 \%$ of last mile links are randomly allocated microwave $(28 \mathrm{GHz})$ technology. \\
\hline$p_{f}$ & $30 \%$ & $30 \%$ of last mile links are randomly allocated fibre-based technology. \\
\hline$N_{i}$ & 10 & Number of iterations per run. \\
\hline$N_{r}$ & 100 & Number of Monte Carlo runs. \\
\hline
\end{tabular}

metres of copper, is $\mathrm{E}\left[T_{v}(r)\right]=\Delta \cdot e^{-\delta \cdot r}$ [4]. The expected capacity of a wireless microwave hop that exhibits line-of-sight (LOS) fading ( $h$ ) conditioned on $r$ is shown below [12, eq. 5]:

$\mathrm{E}\left[T_{m}(\gamma(r)) \mid r\right]=\frac{\mathrm{W}}{\ln (2)} e^{-K} \sum_{n=0}^{\infty} \frac{K^{n}}{n ! \cdot n !} \times G_{2,3}^{3,1}\left[\frac{(1+K)}{\Omega_{r}} \mid \begin{array}{c}0,1 \\ n+1,0,0\end{array}\right]$

where, $K$ is the Rice factor, $W$ is the channel bandwidth in $\mathrm{KHz}, G_{p, q}^{m, n}[\cdot]$ is the Meijer- $G$ function, and $\Omega_{r} \triangleq \mathrm{E}[\gamma \mid r]=$ $P \cdot A \cdot r^{-\alpha} / L_{0} \cdot \sigma^{2}$ is the expectation of the signal-to-noise-ratio $(\gamma)$ conditioned to a particular value of $r$.

The expected delay over a VDSL2 link is mainly determined by the multiplexing/demultiplexing box and is modelled according to the following expression which does not depend on the reach of the copper line [4]:

$$
\mathrm{E}\left[L_{v}\right]=\kappa_{v} \cdot\left(1+1.28 \cdot \lambda_{1} / \lambda_{2}\right) \cdot\left(a_{v}+\epsilon \cdot b_{v}\right)
$$

where, $\left(1+1.28 \cdot \lambda_{1} / \lambda_{2}\right)$ denotes the mean number of small cells served by the designated aggregation point. Moreover, the parameters $\kappa_{v}$ and $a_{v}$ are representative of the generic processing power of the router, and $b_{v}$ represents the router's processing capability with respect to the packet size $\epsilon$. The delay in microwave links is due to two factors: processing and queuing delay $L_{m, 1}$ (4) and the delay caused by the forward-and-retransmit mechanism $L_{m, 2}$. When the data is not correctly detected at the receiving end of the hop (i.e., $\operatorname{Pr}\left([\gamma \mid r]<\tau_{t}\right)$ ), during the technology-specific timeslot $\theta$, it is retransmitted for a number of times before it is declared lost. The waiting period and the retransmission(s) incur an additional delay $L_{m, 2}$; for a wireless link assuming Rician LOS fading it is expressed as follows, where $\mathcal{Q}(\cdot)$ is the Marcum-Q function [12]:

$$
\begin{gathered}
\mathrm{E}\left[L_{m, 2}\right]=\frac{\theta}{\operatorname{Pr}\left([\gamma \mid r]>\tau_{t}\right)} \\
\operatorname{Pr}\left([\gamma \mid r]>\tau_{t}\right)=\mathcal{Q}\left(\sqrt{2 K}, \sqrt{2 \frac{1+K}{\Omega_{r}} \gamma}\right)
\end{gathered}
$$

The fibre-based last mile is considered to have higher capacity than the cells' maximum throughput and lower latency than the lowest $Q_{l}$. Consequently, the measured QoS of users associated with a fibrebased cell are determined by the radio conditions and are never limited by the backhaul.

\section{B. Cost Model}

We propose the following model that captures the cost of backhaul gateways and last mile hops to the small cells, as shown below:

$$
C=\frac{\lambda_{1}}{\lambda_{2}} \sum_{i} p_{i}\left(E_{i}+\mathrm{E}\left[H_{i}(r)\right]\right)=\frac{\lambda_{1}}{\lambda_{2}} \sum_{i} p_{i}\left(E_{i}+\frac{\Gamma\left(\rho_{i} / 2+1\right)}{\left(\pi \lambda_{2}\right)^{\rho_{i} / 2}}\right)
$$

TABLE III

SOLUTION SHORTLIST.

\begin{tabular}{|c|c|l|l|l|}
\hline & Changes & $\begin{array}{l}\text { Tput } \\
(\mathrm{Mbps})\end{array}$ & $\begin{array}{l}\text { Delay } \\
(\mathrm{msec})\end{array}$ & $\begin{array}{l}\text { Cost } \\
\left(10^{6} \$\right)\end{array}$ \\
\hline (i) & $p_{v}=0.05, p_{m}=0.65$ & 1721 & 11.55 & 0.919 \\
\hline (ii) & $p_{v}=0.1, p_{m}=0.4, p_{f}=0.5$ & 1693 & 11.22 & 0.974 \\
\hline (iii) & $p_{v}=0.15, p_{m}=0.05, p_{f}=0.8$ & 1697 & 10.63 & 1.077 \\
\hline (iv) & $p_{v}=0.1, p_{m}=0.6, \lambda=6.5$ & 1697 & 11.16 & 0.371 \\
\hline
\end{tabular}

where, $i=\{m, f\}, E_{i}$ is the equipment cost and $H_{i}(r)=r^{\rho_{i}}$ is the rate of cost increase as a function of distance $r$. The cost calculation is limited to microwave and fibre technologies as no upgrade would require increase in copper-based hops. We assume $E_{m}=\$ 2,000$ and $E_{f}=\$ 50,000$ and $\rho_{m}=\rho_{f}=2$ [13].

\section{Results}

Based on the analytical multi-hop hybrid backhaul performance model, the baseline scenario offers a system capacity expectation of $1137 \mathrm{Mbps}$ and corresponding delay of $12.04 \mathrm{msec}$ (see Figure 3). The corresponding simulation results (100 runs) are shown in Figure 2 and indicate higher throughput (mean $1442.2 \mathrm{Mbps}$ ), albeit, with high users dissatisfaction of $\mathrm{E}\left[\hat{Q}_{c}\right]=171.2 \%$ and $\mathrm{E}\left[\hat{Q}_{l}\right]=173 \%$, on average, with respect to throughput and latency, respectively. Effectively, the backhaul network is overloaded, hence the surge is throughput and deterioration of quality. Priority users are those that associate high weight to the given attribute. There are 10.22 and 14.72 such users with under-par performance re-throughput and latency, respectively.

\section{UPGRADE SOLUTION}

Starting with the baseline scenario described in Table III, we study the effect of replacing VDSL2 links with microwave, increasing the share of fibre links in the last mile, and increasing the density of backhaul gateways in Figures 3 (Top) and (Bottom) for backhaul network capacity and latency, respectively.

\section{A. Shortlisting of potential upgrades}

We set our upgrade target as: the reduction of user dissatisfaction with respect to throughput by $50 \%$. To this end, we aim to increase the backhaul capacity to $1137 \times 1.5=1705.5 \mathrm{Mbps}$. There are four possible upgrades that offer this capacity, as shown in Table IV-A. Based on the performance and cost analytical modelling, two solutions are short-listed for implementation in the simulator. Solution (iii) because it gives the highest improvement, and solution (iv) because it is the cheapest but still delivers second best delay expectation. 

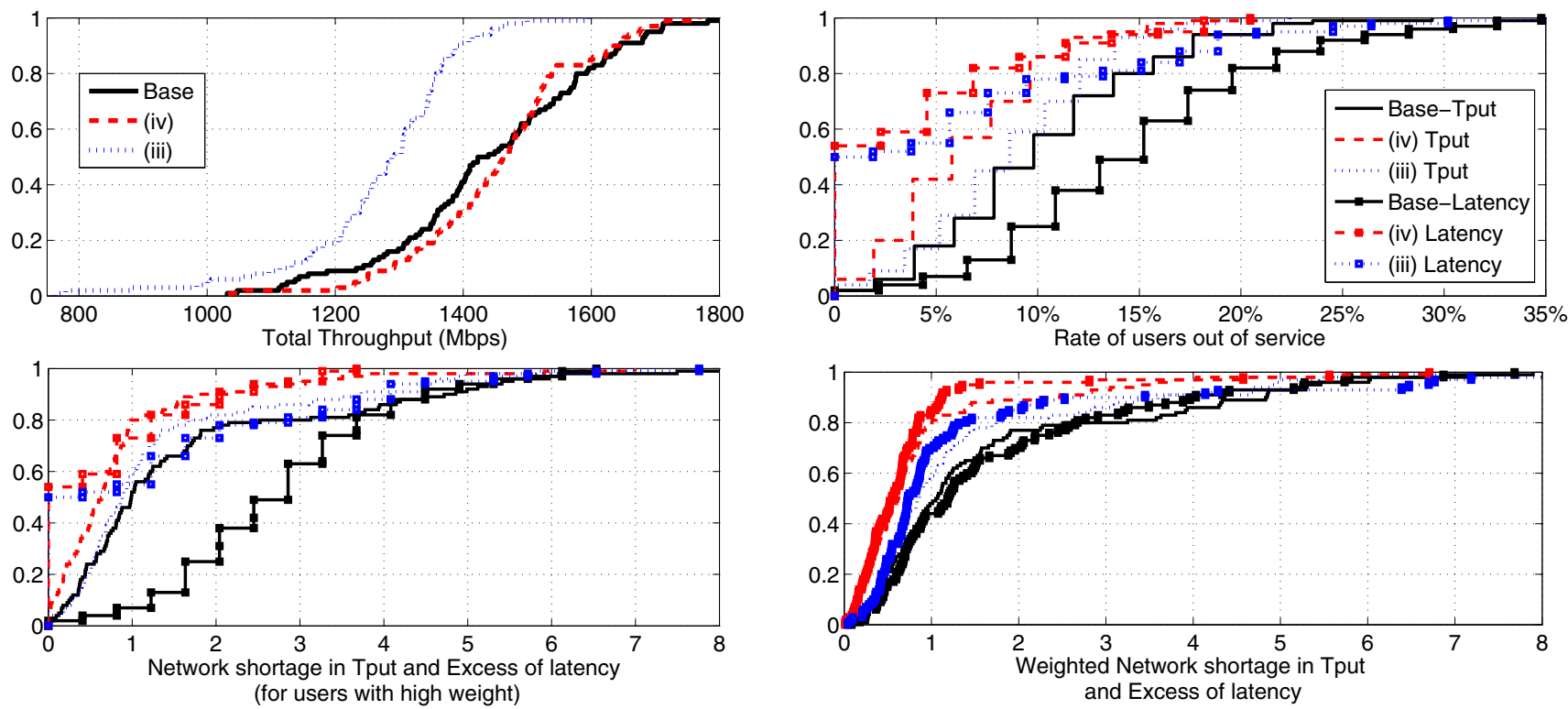

Fig. 2. Simulation results of baseline scenario and potential upgrade solutions. The cumulative distribution function probabilities of each metric are shown over 100 runs on the y-axis.
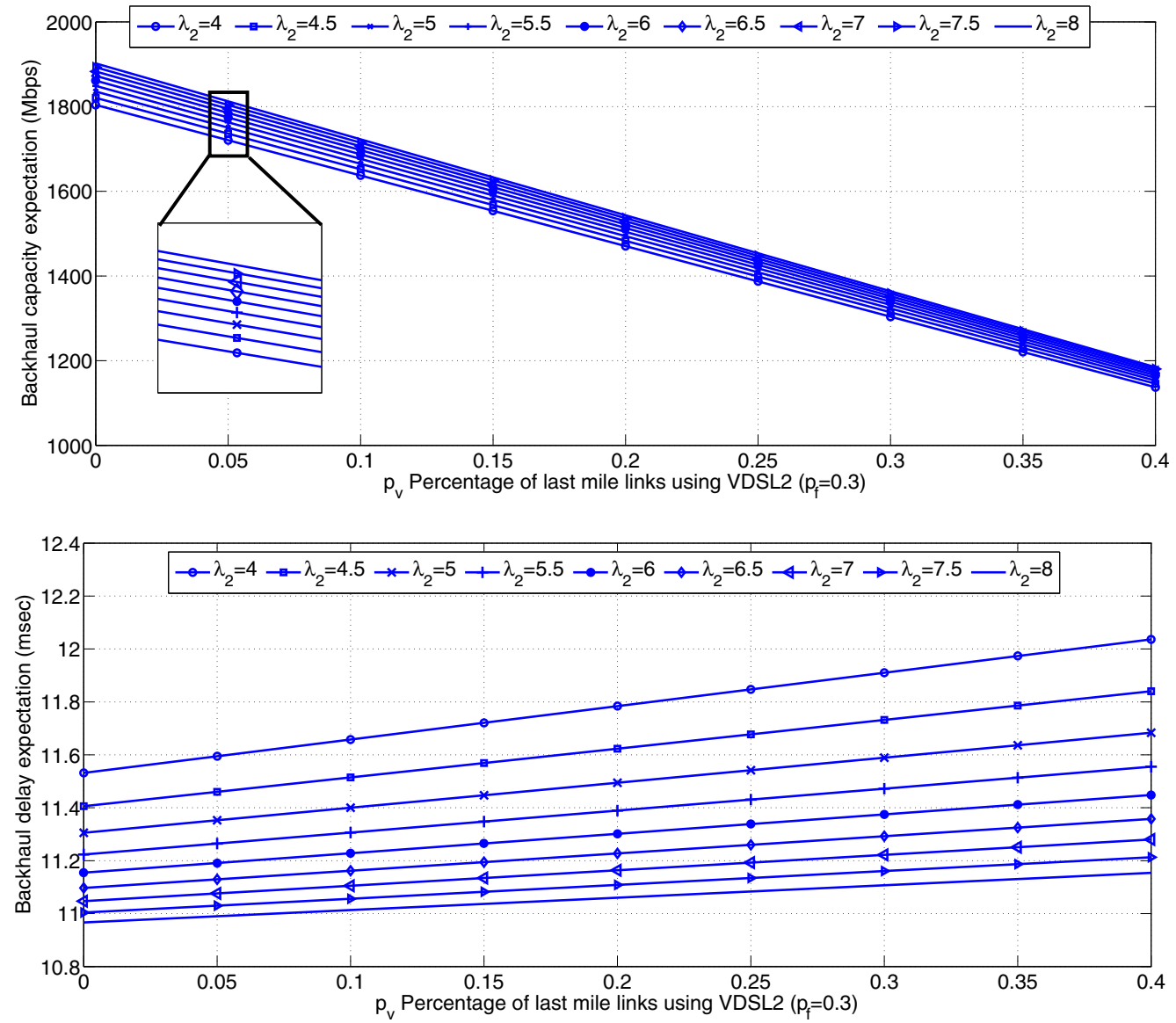

Fig. 3. Effect of key factors on backhaul network capacity and latency. 
TABLE II

PERFORMANCE MODELLING PARAMETERS.

\begin{tabular}{|c|c|l|}
\hline Parameter & Value & Remark \\
\hline$\Delta$ & $105 \mathrm{Mbps}$ & Highest possible throughput over the shortest copper-line reach. \\
\hline$\delta$ & $1.14 \cdot 10^{-3}$ & Throughput decay per unit length of copper. \\
\hline$P$ & $10 \mathrm{dBm}$ & Transmitting power of last mile point-to-point microwave link. \\
\hline$A$ & $43 \mathrm{dBi}$ & Antenna gain on either ends of the microwave link. \\
\hline$L_{0}$ & $120 \mathrm{~dB}$ & Minimum path loss incurred by the the shortest microwave reach. \\
\hline$\alpha$ & 2.5 & Propagation exponent of the microwave link. \\
\hline$f$ & $38 \mathrm{GHz}$ & Operational frequency of the microwave link. \\
\hline$W$ & $112 \mathrm{MHz}$ & Bandwidth of the microwave link channel. \\
\hline$\sigma^{2}$ & $4 \cdot 10^{-15} \mathrm{mw} / \mathrm{KHz}$ & Thermal noise power intensity. \\
\hline$K$ & 10 & Rice factor of the LoS fading in the microwave link channel. \\
\hline$\kappa_{v}, \kappa_{m}$ & 10 & Parameter that reflects the processing power of backhaul gateway [8]. \\
\hline$a_{v}, a_{m}$ & $10 \mu \mathrm{sec}$ & Parameter that reflects the processing power of backhaul gateway [8]. \\
\hline$b_{v}, b_{m}$ & $0.01 \mu \mathrm{sec} / \mathrm{bit}$ & Parameter that reflects the processing power of backhaul gateway re packet size [8]. \\
\hline$\epsilon$ & $1500 \mathrm{bits}$ & Ethernet packet size. \\
\hline
\end{tabular}

TABLE IV

SIMULATION RESULTS.

\begin{tabular}{|c|c|c|c||c|c|c|c|}
\hline Mean & BL & (iv) & (iii) & Mean & BL & (iv) & (iii) \\
\hline$T_{\text {tot }}$ & 1442 & 1455 & 1270 & $\overline{Q_{c}}(\%)$ & 170 & 89 & 136 \\
\hline$O_{c}(\%)$ & 10.22 & 6.4 & 8.57 & $\overline{Q_{l}}(\%)$ & 276 & 68 & 131 \\
\hline$O_{l}(\%)$ & 14.72 & 3.80 & 6.04 & $\hat{Q}_{c}(\%)$ & 171.2 & 90 & 140 \\
\hline & & & & $\hat{Q}_{l}(\%)$ & 173 & 73 & 142 \\
\hline
\end{tabular}

\section{B. Simulation results and final selection}

Each scenario is simulated over 100 Monte Carlo runs, as in Section II-B. The cumulative distribution functions of each metric are shown in Figure 2 and the average values of the results obtained in Table IV-B. Both scenarios (iii) and (iv) succeed in significantly improving the user-centric metrics. In addition, solution (iv) maintains a similar network throughput compared to the baseline, whereas solution (iii) results in $12 \%$ throughput reduction. Nonetheless, solution (iii) targets users with high quality expectations and reduces the number of unsatisfied users (compared to the baseline) by $37 \%$ and $74 \%$ for throughput and latency, respectively. More importantly, the cumulative QoE gap of those users is reduced by $21 \%$ and $52.5 \%$, respectively, at the cost of 0.371 Million Dollars. On the other hand, solution (iv) reduces the QoE gap even further by $46 \%$ and $75 \%$, respectively, in comparison with the baseline, but at a TCO that is almost three times higher than that of solution (iii). Accordingly, money conscious operators would benefit from adopting solution (iii) as a first upgrade to offer better QoE to their existing users and reduce the churn. In contrast, operators that have the means should upgrade using solution (iv) as it offers better quality to users and higher capacity for attracting new users.

\section{CONCLUSION}

We have presented a methodology that successfully identifies the optimum upgrade steps for availing better user QoE and network capacity in future networks that employ a constrained backhaul. The methodology consist of a diagnosis phase, followed by a rectification ranking phase, and a validation phase. The diagnosis and validation are achieved using the UCB scheme, as it pinpoints the network bottlenecks that affect users' perception. The solution ranking is based on the multi-hop hybrid backhaul performance and TCO modelling. Such an analysis offers incumbent networks the possibility to tune their cost expenditures according to their goals, be it retaining their existing users or increasing their network capacity.

\section{ACKNOWLEDGMENT}

The views expressed here are those of the authors and do not necessarily reflect those of the affiliated organisations. The authors would like to thank the UK Engineering and Physical Science Research Council (EPSRC) and BT Research and Innovation for funding this research through an Industrial Cooperative Awards in Science \& Technology (iCASE) studentship. We would also like to acknowledge the support of the University of Surrey 5GIC (http://www.surrey.ac.uk/5gic) members for this work.

\section{REFERENCES}

[1] P. Marshall, "5G Operator survey," tech. rep., The Telecommunications Industry Association (TIA), Jan. 2017.

[2] M. Jaber, N. Kouzayha, Z. Dawy, and A. Kayssi, "On cellular network planning and operation with M2M signalling and security considerations," in IEEE International Conference on Communications Workshops (ICC), pp. 429-434, June 2014.

[3] M. Jaber, M. Imran, R. Tafazolli, and A. Tukmanov, "A distributed SONbased user-centric backhaul provisioning scheme," IEEE Access, vol. PP, no. 99, pp. 1-1, 2016.

[4] M. Jaber, M. Imran, A. Sutton, A. Tukmanov, and R. Tafazolli, "Modular approach for modelling the hybrid multi-hop backhaul performance," IEEE Wireless Communications Letters, vol. 6, no. 2, pp. 262-265, 2017.

[5] H. Beyranvand, W. Lim, M. Maier, C. Verikoukis, and J. Salehi, "Backhaul-aware user association in FiWi enhanced LTE-A heterogeneous networks," vol. 14, pp. 2992-3003, Jun. 2015.

[6] H. Galeana-Zapien and R. Ferrus, "Design and evaluation of a backhaulaware base station assignment algorithm for OFDMA-Based cellular networks," IEEE Transactions on Wireless Communications, vol. 9, pp. 3226-3237, Oct. 2010.

[7] D. Ohmann and G. P. Fettweis, "Minimum duration outage of wireless Rayleigh-fading links using selection combining," in IEEE Wireless Communications and Networking Conference (WCNC), pp. 681-686, Mar. 2015.

[8] G. Zhang, T. Quek, A. Huang, M. Kountouris, and H. Shan, "Delay modeling for heterogeneous backhaul technologies," in Vehicular Technology Conference (VTC Fall), 2015 IEEE 82nd, pp. 1-6, Sept 2015.

[9] B. Berliner, B. Clark, and A. Hartono, "QoS requirements of multimedia applications." [Online], Available: https://cse.osu.edu/, Accessed 26/04/2017, 2011

[10] B. Bangerter, S. Talwar, R. Arefi, and K. Stewart, "Networks and devices for the 5G era," IEEE Communications Magazine, vol. 52, pp. 90-96, Feb. 2014.

[11] R. C. Streijl, S. Winkler, and D. S. Hands, "Mean opinion score (MOS) revisited: methods and applications, limitations and alternatives," Multimedia Systems, vol. 22, no. 2, pp. 213-227, 2016.

[12] N. C. Sagias, G. S. Tombras, and G. K. Karagiannidis, "New results for the Shannon channel capacity in generalized fading channels," IEEE Commun. Lett., vol. 9, pp. 97-99, Feb 2005.

[13] V. Suryaprakash and G. Fettweis, "Modeling backhaul deployment costs in heterogeneous radio access networks using spatial point processes," in 12th International Symposium on Modeling and Optimization in Mobile, Ad Hoc, and Wireless Networks (WiOpt), pp. 725-732, May 2014. 